\title{
The Application of useless Japanese Inventions for Requirements Elicitation in Information Security
}

\author{
Anton Partridge \\ Bournemouth University \\ Poole, UK \\ anton.partridge@me.com
}

\author{
Shamal Faily \\ Bournemouth University \\ Poole, UK \\ sfaily@bournemouth.ac.uk
}

\begin{abstract}
Rules of requirements elicitation in security are broken through the use of Chindōgu, by designing impractical security countermeasures in the first instance, then using these to create usable security requirements. We present a process to conceive the requirements in Chindōgu form. We evaluate the usefulness of this process by applying it in three workshops with data gathered from a European rail company, and comparing requirements elicited by this process with a set of control requirements.
\end{abstract}

\section{Information Security; Requirements Engineering; Creativity; Requirements Elicitation}

\section{INTRODUCTION}

A Chindōgu is defined as "an unusual gadget, originally created as the solution to a particular problem, although effectively it hardly has any utility whatsoever, it's really almost useless" (Mediavilla 2008). A typical Chindōgu is illustrated in Figure 1. Our work aimed to find out if something seemingly useless and impractical as a Chindōgu could be considered viable for solving the wicked problem of information security requirements elicitation. The motivation for using Chindōgu stems from the belief that developing systems which are both usable and secure is as an oxymoron. Security is often seen as a phase of product conception which results in reduced functionality and poor usability. Using Chindōgu as a medium for eliciting requirements in the water industry was found to be successful in the past when applied in (Faily 2012) where the Chindōgu "unintentionally raised awareness of an implicit vulnerability that had been hitherto overlooked".

To gauge the usefulness of Chindōgu for security, we devised a process for eliciting security requirements grounded in the Requirements Engineering literature, and other cases where Chindōgu have been used to solve complex problems. We present our approach in Section 2 before describing our results and lessons learned in Section 3.

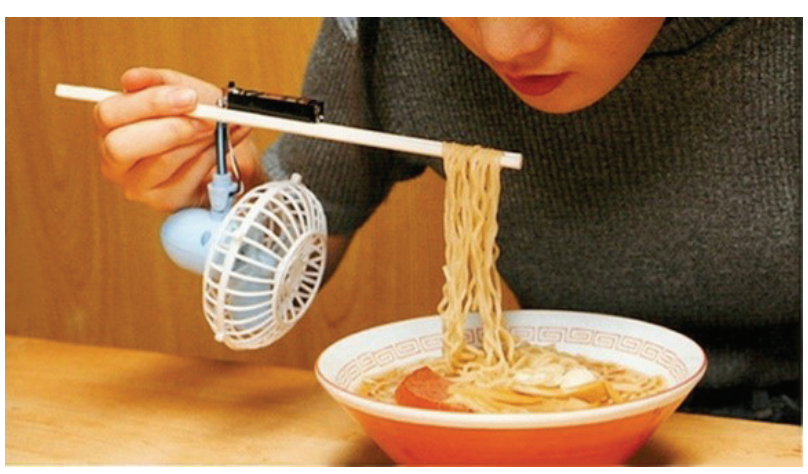

Figure 1: A typical chindōgu (Yoong 2015)

\section{APPROACH}

Our approach for using Chindōgu as a method of security requirements elicitation was grounded in general/creative requirements engineering (RE) practices, such as the use of analogies between the system attributes to other non-computing domains proposed by (Maiden et al. 2004). The creative requirements engineering literature was used to draw on experiences of other novel requirements engineering techniques, together with more conventional requirements engineering practices.

The process was applied as part of the Bournemouth-Athens Network in Critical Infrastructure Security (BANCIS) project. This 


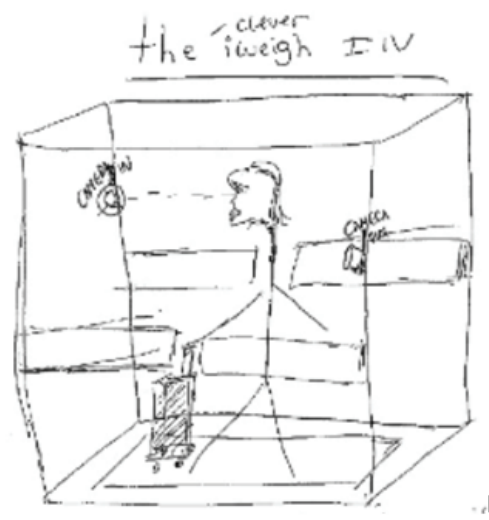

Fed up with thieves at your train station? Why leave it to chance, weigh every visitor on their in and out entry, if they weigh more than they did before entering they may have stolen something!

Your customers weigh in and out, having their picture taken, if they weigh more on their way out an alarm will sound so security will be notified to deal with the thief!

The database connects the picture of the face with customer's weight using facial recondition to ensure accuracy.

Figure 2: A Chindōgu card

is joint partnership between Bournemouth University and Athens University of Economics and Business (AUEB), analysing critical infrastructure and creating exemplars of critical infrastructure scenarios (Faily et al. 2016).

Data was collected from a European rail company to provide problem scenarios for applying the Chindōgu. Once data was gathered, we ran three workshops to apply the process with three participants. This included the first author as a facilitator, and two others with some expertise collecting requirements. Workshops were run in an informal setting with office paraphernalia used to create the Chindōgu, based on an initial sketch, such as that shown in Figure 2. The first workshop was a pilot on a factious scenario to gather feedback and observations before the workshops using problems from the rail company were run. Workshops were then held based on problem data gathered from the rail company. During workshops, interview data from the rail company employeees was analysed, and problems were identified for the Chindōgu process to be run against. Once the Chindōgu and requirements were created, they were specified using the Volere template (Robertson and Robertson 2009), and sent to stakeholders for validation.

To evaluate the Chindōgu derived requirements, a comparison was carried out against control requirements elicited as part of the BANCIS project. The comparison involved the application of predefined metrics on requirements similarity. We evaluated the process in general by observing and interviewing workshop participants. Interview questions concerned the perceived usefulness of a Chindōgu process for eliciting requirements, compared to other requirements elicitation techniques.

\section{RESULTS}

While we found that Chindōgu were perceived to be an effective elicitation technique, an initial learning curve to using the process effectively was observed because participants required prerequisite knowledge to use the process effectively. Workshop participants with a background in security requirements gathering found the process to be an effective way of critically analysing a problem, and helping them think about how the requirements they conceive are not actually an effective solution to a problem from a user's perspective.

Applying the process yielded meaningful security requirements, which were validated by stakeholders at the rail company. Participants were asked to comment on their validity compared to the original problems identified, the feedback indicated that the requirements satisfied the original problem. Although the requirements were valid, the comparison against control requirements from the BANCIS project highlighted some missing requirements; these were concerned the operation of subsystems which would be affected by a high-level requirement.

\section{CONCLUSION}

We found that Chindōgu provide a means to quickly elicit high-level security requirements based on problems identified in interviews with stakeholders. Requirements elicited through this process did, however, need further analysis to establish their implications to other security systems and requirements. Nonetheless, the use of Chindōgu appears to be a powerful tool for engaging stakeholders.

The impracticalities of the Chindōgu inventions encouraged workshop participants to think critically about the requirements conceived. This, together with the unfamiliarity of the process, helped participants abandon preconceptions, thereby aiding creative thinking.

\section{ACKNOWLEDGEMENTS}

The research described in this paper was partially funded by Fusion Investment Funded BournemouthAthens Network in Critical Infrastructure Security.

\section{REFERENCES}

Faily, S. (2012). Analysing chindōgu: Applying defamiliarisation to security design. $\mathrm{CHI} 2012$ 
Workshop on Defamiliarisation in Innovation and Usability.

Faily, S., Lykou, G., Partridge, A., Gritzalis, D., Mylonas, A., and Katos, V. (2016). HumanCentered Specification Exemplars for Critical Infrastructure Environments. In Proceedings of the 30th British $\mathrm{HCl}$ Group Annual Conference on People and Computers. To Appear.

Maiden, N., Gizikis, A., and Robertson, S. (2004). Provoking creativity: imagine what your requirements could be like. IEEE Software, 21(5):68-75.
Mediavilla, A. R. (2008). Chindogu: The Art Of The Unuseless. http://www.curiosite.com/scripts/ news/ennews.php?frmldPagina $=10077$.

Robertson, J. and Robertson, S. (2009). Volere Requirements Specification Template: Edition 14 - January 2009. http://www.volere.co. uk/template.htm.

Yoong, S. (2015). Chindogu: Only in Japan will these things exist.... http://www.theculturemap.com/ chindogu-only-in-japan-things-exist/. 